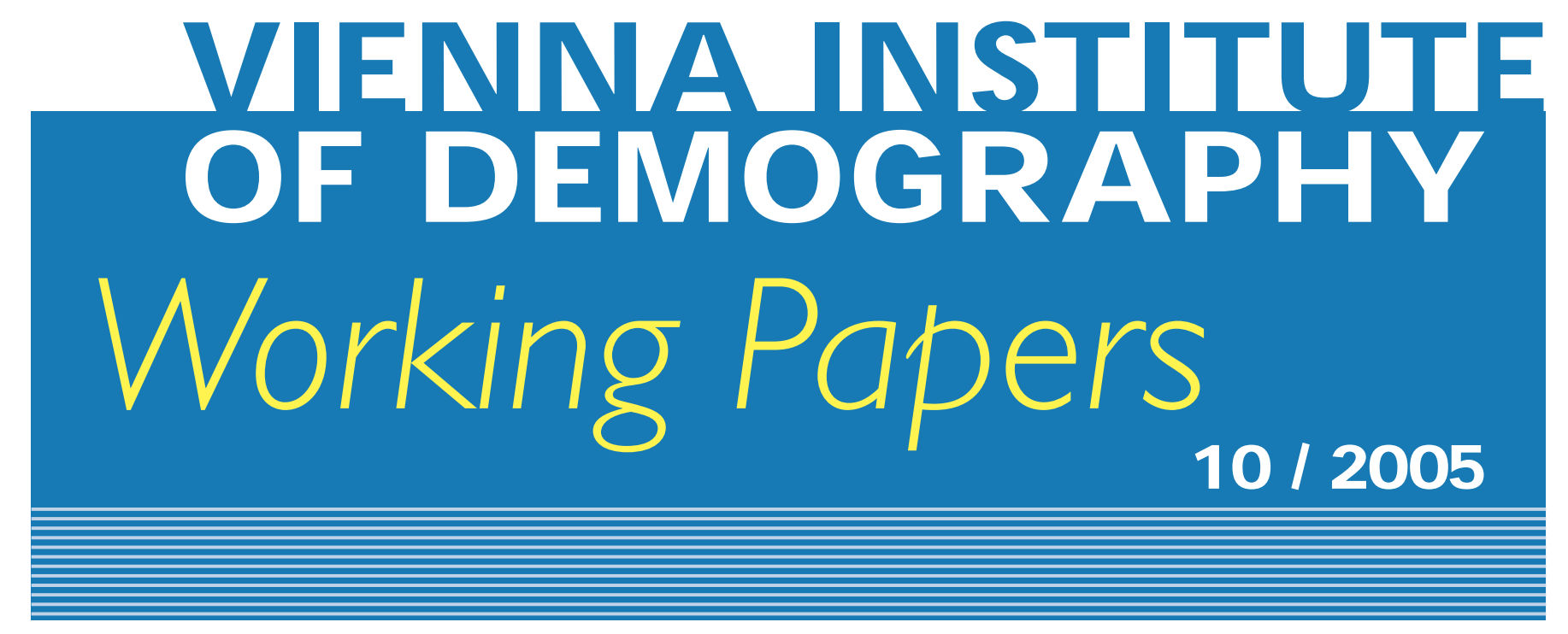

Dalkhat M. Ediev

\title{
Extension of Fisher's Classical Result on Exponential Dynamics of the Reproductive Value to a Wide Class of Populations
}

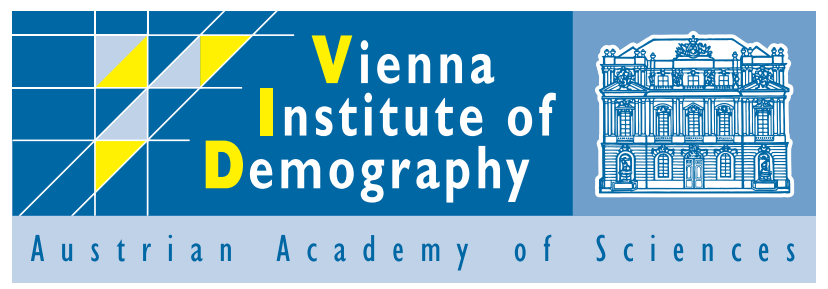

Vienna Institute of D emography A ustrian A cademy of Sciences

Prinz Eugen-Straße 8-10 · A-1040 Vienna · A ustria

E-Mail: vid@ oeaw.ac.at

W ebsite: www.oeaw.ac.at/vid 


\begin{abstract}
The classical result by R.A. Fisher concerning reproductive value dynamics is expanded to the case of varying vital rates with a constant cohort Lotka's $r$. Based on the demographic potential approach, generalization of the concept of reproductive value is introduced, which exhibits exponential dynamics both in the classical case of constant vital rates and in a wider class of populations. The generalized reproductive value introduced in the paper fits the classical interpretation by R.A. Fisher as a discounted sum of future births in the general class of models addressed in the paper. Results could be of importance for estimating the fitness of biological populations, aggregate population modeling, and studying the long-term consequences of varying vital rates.
\end{abstract}

\title{
Keywords
}

Reproductive value, Lotka's $r$, Malthusian parameter, demographic potential, fitness

\section{Authors}

Dalkhat M. Ediev is a research scholar at the Vienna Institute of Demography of the Austrian Academy of Sciences, and docent of the Mathematics Chair at the KarachayCherkessian State Technological Academy, Russia.

\section{Acknowledgements}

The work was supported in part by the Russian Foundation for Fundamental Research under Grant \#05-06-80432 "Development of mathematical models and methods for estimating the reproduction characteristics of a small-size population". 


\title{
Extension of Fisher's classical result on exponential dynamics of the reproductive value to a wide class of populations
}

\author{
Dalkhat M. Ediev
}

\section{Introduction}

Studying populations with constant fertility and survivorship functions, R.A. Fisher (1930) showed that the change rate of the population's reproduction value $V(t)$ is always equal to the Lotka's $r$, no matter how disturbed from the stable state the population age structure is. Apart from theoretical applications, this result is important for estimating Lotka's $r$ based on observed dynamics of population structure in the absence of direct data on vital rates and for aggregate population forecasting in the long run. Here we strengthen this result showing that the property of the aggregate reproductive value proven by R.A. Fisher for the case of constant reproduction regime can be expanded to time-varying population models with constant cohorts' Lotka's $r$.

We base our study on the demographic potential approach, which enables generalization of the reproductive value concept to arbitrary population models and facilitates studying long-term population dynamics.

\section{Reproductive value and demographic potential}

R.A. Fisher introduced the concept of reproductive value as the present value of future births from a person discounted by the Lotka's $r$ :

$$
v(x)=\int_{x}^{\infty} \frac{l(y)}{l(x)} f(y) e^{-r(y-x)} d y,
$$

where $v(x)$ stands for the reproductive value of a person aged $x, l(x)$ is the survivorship function, i.e., the accumulated probability to survive from the birth to the age $x$, and $f(x)$ is the fertility function. Lotka's $r$ is defined in the common way and satisfies the equality:

$$
\int_{0}^{\infty} l(x) f(x) e^{-r x} d x=1 .
$$

This implies, in particular, that the newborn's reproductive value is set to be one, $v(0)=1$.

It was proven by R.A. Fisher that the change rate of the population's total reproductive value is equal to the Lotka's $r$ :

$$
\frac{1}{V(t)} \cdot \frac{d}{d t} V(t) \equiv r
$$

Here $V(t)=\int_{0}^{\infty} n(x ; t) v(x) d x$ is the total reproductive value of the population at time $t$, where 
$n(x ; t)$ is the population density at age $x$ at calendar time $t$. This property is convenient for demographic analysis and population modeling, and we are interested in expanding it to a wider class of population models.

Naturally, the reproductive value concept can be introduced in the presented way for time-independent reproduction regimes only. For the general case, however, another concept can be introduced, which allows generalizing Fisher's results (Ediev, 2003a):

$$
c(x, t)=\int_{x}^{\infty} \frac{l(y, t)}{l(x, t)} f(y, t) c(0, t+y) d y .
$$

Here $c(x, t)$ is the demographic potential of person born at time $t$, at his age $x$. Other functions in (4) are similar to those in (1), (2) with the second variable being the time of birth. In other words, functions in (4) are explicitly written as functions of cohorts.

Demographic potential reflects relative impact of a person on long-term population dynamics. The most remarkable feature of the total demographic potential of the population is that it always equals to some constant depending on original population structure only. Indeed, the derivative of the total demographic potential of any population following the time-dependent reproduction regime imputed in (4) is equal to zero:

$$
\begin{aligned}
& \frac{d C(t)}{d t}=\frac{d}{d t} \int_{0}^{\infty} n(x ; t) c(x, t-x) d x= \\
& =\frac{d}{d t} \int_{0}^{\infty} B(t-x) l(x, t-x) \int_{x}^{\infty} \frac{l(y, t-x)}{l(x, t-x)} f(y, t-x) c(0, t-x+y) d y d x= \\
& =\frac{d}{d t} \int_{0}^{\infty} B(t-x) \int_{x}^{\infty} l(y, t-x) f(y, t-x) c(0, t-x+y) d y d x= \\
& =\frac{d}{d t} \int_{-\infty}^{t} B(z) \int_{t-z}^{\infty} l(y, z) f(y, z) c(0, z+y) d y d z= \\
& =B(t) \int_{0}^{\infty} l(y, t) f(y, t) c(0, t+y) d y-\int_{-\infty}^{t} B(z) l(t-z, z) f(t-z, z) c(0, t) d z= \\
& =B(t) c(0, t)-c(0, t) \int_{0}^{\infty} n(x ; t) f(x, t-x) d x=B(t) c(0, t)-c(0, t) B(t)=0 .
\end{aligned}
$$

Here $C(t)$ is the total demographic potential of the population and $B(t)$ is the birth rate at time $t$.

The concept of reproductive value could be generalized to the arbitrary population model in the following way:

$$
v(x ; t)=\frac{c(x ; t)}{c(0, t)}=\frac{c(x, t-x)}{c(0, t)} .
$$

We separate by comma the time of the birth for cohorts and use the semicolon to separate the calendar time variable from the variable of age. Hence, $c(x, t)$ and $v(x, t)$ state for 
functions of the cohort born at time $t$, while $c(x ; t)$ and $v(x ; t)$ state for the demographic potential and the generalized reproductive value of a person aged $x$ at time $t$.

When reproduction regime becomes independent of time, generalized reproductive values (6) turn into Fisher's reproductive values (1). One could note that the reproductive value of newborn is always equal to unity, i.e., generalized reproductive values reflect relative long-term demographic impacts of people living at the same time with newborn's impact taken as a measuring unit.

\section{Dynamics of the generalized reproductive value in populations with varying reproduction rates and constant cohort Lotka's $r$}

Let the population vital rates vary at arbitrary manner with only one restriction imposed, the constancy of the Lotka's $r$ for cohorts:

$$
\int_{0}^{\infty} l(x, t) f(x, t) e^{-r x} d x \equiv 1 .
$$

Note that this does not imply constancy of either the period Lotka's $r$, the net reproduction rates or of the generation lengths.

The main equation for the demographic potentials (4) can be written for the newborns' potential in the following form of the renewal equation:

$$
c(0, t)=\int_{0}^{\infty} l(x, t) f(x, t) c(0, t+x) d x .
$$

The ergodic property similar to that of population models (Arthur, 1982) holds for the demographic potentials dynamics with reversed time (Ediev, 2003a), which implies that the equation (8) has only a single set of solutions, differing only by a constant multiplier. This set of solutions can be derived explicitly in the case of constant Lotka's $r$. Indeed, (8) is equivalent to the following equation:

$$
c(0, t) e^{r t}=\int_{0}^{\infty} l(x, t) f(x, t) e^{-r x} c(0, t+x) e^{r(t+x)} d x .
$$

Given equation (7) one can easily check that the following non-trivial solution satisfies equation (9):

$$
c(0, t)=c(0,0) e^{-r t}
$$

with arbitrary non-zero constant $c(0,0)$, which is the demographic potential of a newborn at time 0 .

Now, turning to the total reproductive value of the population, it is easy to establish that its change rate is equal to the Lotka's $r$ : 


$$
\begin{gathered}
V(t)=\int_{0}^{\infty} n(x ; t) v(x ; t) d x=\int_{0}^{\infty} n(x ; t) \frac{c(x ; t)}{c(0, t)} d x= \\
=\frac{1}{c(0, t)} C(t)=\frac{e^{r t}}{c(0,0)} C(0)=V(0) e^{r t} .
\end{gathered}
$$

Hence, the result shown by R.A. Fisher for the time-independent reproduction regimes can be expanded to varying reproduction regimes with constant Lotka's $r$ (calculated for cohorts rather than for periods).

Using relations (4), (6), and (10) it is also possible to show that Fisher's interpretation of the reproductive value as the discounted present value of future births holds in the case addressed here:

$$
\begin{aligned}
& v(x ; t)=\frac{c(x, t-x)}{c(0, t)}=\int_{x}^{\infty} \frac{l(y, t-x)}{l(x, t-x)} f(y, t-x) \frac{c(0, t-x+y)}{c(0, t)} d y= \\
& =\int_{x}^{\infty} \frac{l(y, t-x)}{l(x, t-x)} f(y, t-x) e^{-r(y-x)} d y .
\end{aligned}
$$

Note that all functions in the last integral relate to those aged $x$ at time $t$.

\section{Implications}

Several implications of the results obtained can be pointed out. Firstly, the results suggest the importance of cohort rates in long-term population dynamics. Despite any fluctuations in observed rates for calendar periods, these are cohort rates, which determine the longterm population dynamics. Given the higher stability of the cohort rates compared to the period rates, this implies more stability of population dynamics in the long run. Traditionally, the intrinsic rate of population change implied by the Lotka's $r$ (or the Malthusian parameter) was widely used as a measure of fitness in evolutionary studies (Fisher, 1930; Haldane 1932; Wright, 1968; Schaffer, 1974, 1981; Caswell, 1980, 1981; Yodzis, 1981). Classical case of constant vital rates, which essentially underlines such an approach, is obviously not in accordance with real population dynamics. However, if Lotka's $r$ for population cohorts is constant and, in particular, is not affected by environmental changes affected buy the population itself, then the result obtained here suggests that the cohorts' Malthusian parameter is indeed a measure of fitness. Even more, the reproductive value obtained in the way suggested by R.A. Fisher as a sum of future births discounted by the Lotka's $r$, will still reflect the long-term share of a person in the population's gene pool. It is the cohort Lotka's $r$, which should be used for discounting the births, however, even though this parameter may be irrelevant for actual population dynamics in the nearest future.

Results obtained here provide a basis for estimating the unknown value of Lotka's $r$ based on the dynamics of the population age structure. Indeed, in order to do this one needs to know age-specific reproductive values. These values, in turn, depend on vital rates and, in particular, on Lotka's $r$ itself. Hence, the task of estimating Lotka's $r$ based on the 
dynamics of the population's reproductive value seems to be cumbersome. However, studying real populations and making simulations demonstrate that applying some approximate age profile of reproductive values close to the unknown actual profile could result in good estimates of the dynamics of the aggregate reproductive value (Ediev, 2003a). This approach can be improved by applying the iterative procedure with estimates of Lotka's $r$ used to improve approximations for the reproductive values. The technique of estimating Lotka's $r$ could be of special importance when data on age-specific mortality are available, which may sufficiently improve approximation of reproductive values. In particular, such a situation can arise in studies of small populations and in non-human populations.

The exponential growth property of the reproductive value obtained here is helpful in studying long-term consequences of various changes in survivorship and fecundity. If these changes do not affect Lotka's $r$ of cohorts, the dynamics of the reproductive value will not be affected either. The ratio of the reproductive value to the birth rate equals the mean age at childbearing in the stable population (e.g., Keyfitz, 2005, p. 186). Hence, the long run birth rate trend will be close to the product of the reproductive value (which grows exponentially) by the mean age at childbearing in the stable equivalent population. If, in particular, the initially stable population experiences transition to a new time-independent reproduction regime while keeping the cohort Lotka's $r$ constant, the final effect of the transition on the birth rate will be proportional to change in the mean age at childbearing in the population. This result was also obtained elsewhere using different approach (Goldstein and Schlag, 2003). One particular case of such dynamics is that of constant net reproduction rate and constant mean age at childbearing combined with changes in the variance of the fecundity function. In this case the dynamics of the reproductive value and of the population size will in the long run not be affected by changes in the shape of the fecundity function.

The exponential dynamics property of the reproductive value was used in developing aggregate models for the population long-term dynamics (Ediev, 2001, 2003a). The main idea of the approach is to decompose the aggregate population change into two components. First component is the general effect of the reproduction regime, which is reflected in the dynamics of the reproductive value. Another component is the populationspecific effect of changes in the age structure, which is reflected in the dynamics of the average reproductive value. Results obtained here suggest effectiveness of this approach to aggregate population modeling with cohort Lotka's $r$ used as an input parameter of the model. The approach will also be facilitated by the fact that cohort indicators of completed fertility are usually more stable than period indicators.

The final implication, which we would like to outline, concerns the problem of measuring the convergence of age structures of populations following the same reproduction regimes. Appropriate measures of such convergence allow someone to monitor the processes of demographic convergence and to test hypotheses about similarity of reproduction regimes of different populations taking into account the age structure's dynamics (i.e. without direct data on vital rates). In the case of studying the convergence of single population's age structure to the structure of stable population under the assumption of constant vital rates, the measure of convergence originated from the Kullback-Leibler 
distance of information theory was proposed by Tuljapurkar, Schoen and Kim (Tuljapurkar, 1982; Schoen and Kim, 1991, 1993). This measure monotonically decreases as the population's age structure converges to the stable asymptote. Hence, studying the direction and rate of change of the Kullback-Leibler distance would-theoretically-enable testing the hypothesis about the direction of population's convergence (i.e., in fact, about the reproduction regime it follows). Unfortunately, this measure cannot be applied to a more realistic case of varying vital rates, and also not to measuring the convergence of two non-stable populations. Even more important, it is sensitive to errors in input data, which confines its practical applicability. However, a general class of monotonic measures of convergence can be introduced, which includes as special cases the Kullback-Leibler distance and other-more robust-measures (Ediev, 2003b, 2004). In particular, in the general case of varying vital rates the following measures of populations age structures' convergence have been proved to decrease monotonically:

$$
D_{t}=\sum_{x} c_{x ; t} d\left(\frac{N^{1}{ }_{x ; t}}{C^{1}}, \frac{N^{2}{ }_{x ; t}}{C^{2}}\right),
$$

this expression is equivalent to expression (23) at p. 44 of the aforementioned work. Here the discrete population model is concerned, $c_{x ; t}$ and $N_{x ; t}$ stand for the demographic potential and the population stock at age $x$ at calendar time $t$; superscripts " 1 " and "2" denote population numbers of two populations to be compared; and $d\left(N^{1}{ }_{x ; t}, N^{2}{ }_{x ; t}\right)$ is any convex and homogeneous function of its variables, e.g., the absolute value of their difference, $d\left(N_{x ; t}^{1}, N_{x ; t}^{2}\right)=\left|N_{x ; t}^{1}-N_{x ; t}^{2}\right|$. Demographic potentials are put into denominators in (13) in order to guarantee similar asymptotic dynamics of the populations compared. The main drawback of measure (13) is the necessity to estimate demographic potentials dynamics $c_{x ; t}$. When the cohort Lotka's $r$ is constant, however, results obtained here allow substitution of absolute demographic potentials by reproductive values, which are easier to approximate in practical studies:

$$
\begin{aligned}
& D_{t}=\sum_{x} \frac{c_{x ; t}}{c_{0 ; t}} c_{0 ; t} d\left(\frac{N^{1}{ }_{x ; t}}{C^{1}}, \frac{N^{2} x ; t}{C^{2}}\right)=\sum_{x} v_{x ; t} d\left(c_{0 ; t} \frac{N^{1}{ }_{x ; t}}{C^{1}}, c_{0 ; t} \frac{N^{2}{ }_{x ; t}}{C^{2}}\right)= \\
& =\sum_{x} v_{x ; t} d\left(\frac{N^{1}{ }_{x ; t}}{V^{1}{ }_{t}}, \frac{N^{2}{ }_{x ; t}}{V^{1}{ }_{t}}\right)=e^{-r t} \sum_{x} v_{x ; t} d\left(\frac{N^{1}{ }_{x ; t}}{V^{1}{ }_{0}}, \frac{N^{2}{ }_{x ; t}}{V^{1}{ }_{0}}\right) \approx e^{-r t} \sum_{x} v^{*}{ }_{x} d\left(\frac{N^{1}{ }_{x ; t}}{V^{1}{ }_{0}}, \frac{N^{2}{ }_{x ; t}}{V^{1}{ }_{0}}\right),
\end{aligned}
$$

where $v^{*}{ }_{x}$ is some approximate profile of reproductive values. Each of last three expressions in (14) could be used in study of the populations' convergence, depending on data available. In particular, the following measures of dissimilarity of age structures of two populations will decrease monotonically as long as the populations follow the same reproduction regime with cohort Lotka's $r$ being constant:

$$
\begin{aligned}
D^{1}{ }_{t} & =\sum_{x}\left|\frac{v_{x ; t} N^{1}{ }_{x ; t}}{V^{1}{ }_{t}}-\frac{v_{x ; t} N^{2}{ }_{x ; t}}{V^{2}{ }_{t}}\right| \approx e^{-r t} \sum_{x} v^{*} x\left|\frac{N^{1}{ }_{x ; t}}{V^{1}{ }_{0}}-\frac{N^{2}{ }_{x ; t}}{V^{2}{ }_{0}}\right|, \\
D^{k}{ }_{t} & =\sum_{x} \sqrt{\left|\left(\frac{v_{x ; t} N^{1}{ }_{x ; t}}{V^{1}{ }_{t}}\right)^{k}-\left(\frac{v_{x ; t} N^{2}{ }_{x ; t}}{V^{2}{ }_{t}}\right)^{k}\right|} \approx e^{-r t} \sum_{x} v^{*} \sqrt[k]{\left|\left(\frac{N^{1}{ }_{x ; t}}{V^{1}{ }_{0}}\right)^{k}-\left(\frac{N^{2}{ }_{x ; t}}{V^{2}{ }_{0}}\right)^{k}\right|}, k \geq 1,
\end{aligned}
$$




$$
\begin{aligned}
D^{\ln }{ }_{t}= & \sum_{x=0}^{X}\left(\frac{v_{x ; t} N^{1}{ }_{x ; t}}{V^{1}{ }_{t}}-\frac{v_{x ; t} N^{2}{ }_{x ; t}}{V^{2}{ }_{t}}\right) \ln \left(\frac{V^{1}{ }_{t} v_{x ; t} N^{2}{ }_{x ; t}}{v_{x ; t} N^{1}{ }_{x ; t} V^{2}{ }_{t}}\right) \approx \\
& \approx e^{-r t} \sum_{x} v^{*}{ }_{x}\left(\frac{N^{1}{ }_{x ; t}}{V^{1}{ }_{0}}-\frac{N^{2}{ }_{x ; t}}{V^{2}{ }_{0}}\right) \ln \left(\frac{V^{2}{ }_{0} N^{1}{ }_{x ; t}}{N^{2}{ }_{x ; t} V^{1}{ }_{0}}\right), \\
D^{*}{ }_{t} & =\sum_{x=0}^{X}\left(\frac{v_{x ; t} N^{1}{ }_{x ; t}}{V^{1}{ }_{t}}-\frac{v_{x ; t} N^{2}{ }_{x ; t}}{V^{2}{ }_{t}}\right)\left(1-\frac{V^{1}{ }_{t} v_{x ; t} N^{2}{ }_{x ; t}}{v_{x ; t} N^{1}{ }_{x ; t} V^{2}{ }_{t}}\right) \approx \\
& \approx e^{-r t} \sum_{x} v^{*}{ }_{x}\left(\frac{N^{1}{ }_{x ; t}}{V^{1}{ }_{0}}-\frac{N^{2}{ }_{x ; t}}{V^{2}{ }_{0}}\right)\left(1-\frac{V^{1}{ }_{0} N^{2}{ }_{x ; t}}{N^{1}{ }_{x ; t} V^{2}{ }_{0}}\right) .
\end{aligned}
$$

Observing dynamics of these indexes it is possible to test if different populations follow the same reproduction regime and-based on the value of distances (15)-(18)-to infer how long the populations have followed similar reproduction regimes. The same measures could also be used for testing if the population dynamics is consistent with the reproduction regime supposed to be followed by the population. 


\section{References}

Arthur, W.B., 1982. The Ergodic Theorems of Demography: a Simple Proof. Demography $19,439-445$.

Caswell, H., 1980. On the Equivalence of Maximizing Reproductive Value and Maximizing Fitness. Ecology 61, 19-24.

Caswell, H., 1981. Reply to comments by Yodzis and Schaffer. Ecology 62, 1685.

Ediev, D.M., 2001. Aggregate Population Forecasting With the Use of Demographic Potentials Technique. Investigated In Russia (Demographic section). 38e, 408-431. (http://zhurnal.ape.relarn.ru/articles/2001/038e.pdf)

Ediev, D.M., 2003a. The Concept of Demographic Potential and Its Applications. Matematicheskoye Modelirovanie (Mathematical modeling). 15 (12), 37-74.

Ediev, D.M., 2003b. On Monotonic Convergence to Stability. Demographic Research 8 31-60. (http://www.demographic-research.org/volumes/vol8/2/8-2.pdf)

Ediev, D.M., 2004. On Comparing the Age Structures of Real Populations. Voprosy Statistiki (Statistical Studies) 10, 16-27.

Fisher, R.A., 1930. The genetical theory of natural selection. Dover Publications, New-York.

Goldstein, J.R., Schlag, W., 2003. Longer life and population growth. Population and Development Review 25, 741-747.

Haldane, J.B.S., 1932. The Causes of Evolution. Harper \& Broth, New York.

Keyfitz, N., 2005. Applied Mathematical Demography. $3^{\text {rd }}$ edition. Springer Science+Business Media, New York.

Schaffer, W.M., 1974. Selection for optimal life histories: the effects of age structure. Ecology 55, 291-303.

Schaffer, W.M., 1981. On reproductive value and fitness. Ecology 62, 1683-1685.

Schoen, R., Kim, Y.J., 1991. Movement toward stability as a fundamental principle of population dynamics. Demography 28, 455-466.

Schoen, R., Kim, Y.J., 1993. On the intrinsic force of convergence to stability. Mathematical population studies. 4, 89-102.

Wright, S., 1968. Evolution and the Genetics of Populations, vol. 1: Genetic and Biometric Foundations. Univ. Chicago Press, Chicago. 
Tuljapurkar, S.D., 1982. Why use population entropy? It determines the rate of convergence. J. of Math. Biol. 13, 325-337.

Yodzis, P. 1981. Concerning the sense in which maximizing fitness is equivalent to maximizing reproductive value. Ecology 62, 1681-1682. 


\section{VIENNA INSTITUTE OF DEMOGRAPHY}

\section{Working Papers}

Dalkhat M. Ediev. Long-Term Effects of Childbearing Postponement. VID Working Papers 09/2005. Vienna: Vienna Institute of Demography.

Dimiter Philipov, Zsolt Spéder, and Francesco C. Billari. Now or Later? Fertility Intentions in Bulgaria and Hungary and the Impact of Anomie and Social Capital. VID Working Papers 08/2005. Vienna: Vienna Institute of Demography.

Franz Schwarz. Widening Educational Inequalities in Mortality: An Analysis for Austria with International Comparisons. VID Working Papers 07/2005. Vienna: Vienna Institute of Demography.

Kim, Jungho, Henriette Engelhardt, Alexia Prskawetz, and Arnstein Aassve. Does Fertility Decrease the Welfare of Households? An Analysis of Poverty Dynamics and Fertility in Indonesia. VID Working Papers 06/2005. Vienna: Vienna Institute of Demography.

Kim, Jungho. Women's Education in the Fertility Transition: The Reversal of the Relationship Between Women's Education and Birth Spacing in Indonesia. VID Working Papers 05/2005. Vienna: Vienna Institute of Demography.

Kim, Jungho. Learning by Doing and Learning from Others in Contraceptive Technology. VID Working Papers 04/2005. Vienna: Vienna Institute of Demography.

Heiland, Frank, Alexia Prskawetz, and Warren C. Sanderson. Do the MoreEducated Prefere Smaller Families? VID Working Papers 03/2005. Vienna: Vienna Institute of Demography.

Henriette Engelhardt und Alexia Prskawetz. Arbeitsmarkt und Demographie. VID Working Papers 02/2005. Vienna: Vienna Institute of Demography.

Sobotka, Tomáš, Maria Winkler-Dworak, Maria Rita Testa, Wolfgang Lutz, Dimiter Philipov, Henriette Engelhardt, and Richard Gisser. Monthly Estimates of the Quantum of Fertility: Towards a Fertility Monitoring System in Austria. VID Working Papers 01/2005. Vienna: Vienna Institute of Demography. 